\title{
Loop-freeness in multipath BGP through propagating the longest path
}

\author{
Iljitsch van Beijnum \\ IMDEA Networks \\ iljitsch.vanbeijnum@imdea.org
}

\author{
Jon Crowcroft \\ University of Cambridge \\ Computer Laboratory \\ Jon.Crowcroft@cl.cam.ac.uk
}

\author{
Francisco Valera and Marcelo Bagnulo \\ Universidad Carlos III de Madrid \\ fvalera@it.uc3m.es, marcelo@it.uc3m.es
}

\begin{abstract}
The concurrent use of multiple paths through a communications network has the potential to provide many benefits, including better utilisation of the network and increased robustness. A key part of a multipath network architecture is the ability for routing protocols to install multiple routes over multiple paths in the routing table. In this paper we propose changes to local BGP processing that allow a BGP router to use multiple paths concurrently without compromising loop-freeness.
\end{abstract}

Index Terms-Routing, BGP, multipath, loop-freeness

\section{INTRODUCTION}

USING multiple paths concurrently to send packets to a single destination has a number of advantages. In a multipath-aware network, there are fewer instances where available links remain unused because no traffic is routed over them. It also reduces the need to manually optimise traffic flow (traffic engineering). Additional benefits can be gained if transport protocols can be made aware of the multiple paths and direct flows or sub-flows over different paths. In that case, users gain better robustness because the reaction to failures for a subset of the available paths to a destination can be handled at transport time scales, which tend to be much shorter than routing time scales, especially in the case of inter-domain routing [1]. It also allows for dynamic adjustment to congestion [1].

However, in order to make use of multiple paths, it is necessary for routing protocols to be multipath-aware; traditionally, routing protocols only select a single, best path to any given destination, but common IP routing protocols already support the use of multiple paths in either their design or implementation within certain limitations. OSPF [2] supports equal-cost multipath: the sum of the metrics (costs) of the links that are part of each path must be equal. EIGRP [3] is capable of utilising multiple paths with differing costs. Although not part of the specification, many BGP [4] implementations are capable of utilising multiple equal-cost paths concurrently. The limitation that almost all of the properties of the different paths must be equal makes sure that using multiple paths will not lead to loops, but is overly conservative and suppresses loop-free additional paths.
We propose changes to BGP's path selection and path dissemination rules that allow for the use of a much wider selection of paths concurrently without compromising loopfreeness. Because a router running BGP tends to receive multiple paths to the same destination from different neighbouring routers, the modifications to allow for the use of multiple paths can be limited to each individual router and modifications to the BGP protocol are unnecessary. A modified router first applies normal BGP policy criteria and then selects a subset of the received paths for concurrent use. We then disseminate the path with longest AS_PATH length to upstream ASes. Although disseminating a path that has a larger number of ASes in its AS_PATH seems counterintuitive, it has the property of allowing the router to use all paths with a smaller or equal AS_PATH length without risking loops.

However, this change has the implication that there is no longer a one-to-one relationship between the paths that packets follow through the network and the path that is advertised in BGP. The resulting obfuscation of the network's topology as seen by observers at the edge can either be considered harmful, for those who want to study networks or apply policy based on the presence of certain intermediate domains, or useful, for those intent on hiding the inner workings of their network.

Our multipath BGP modifications allow individual ASes to deploy multipath BGP and gain its benefits without coordination with other ASes. Hence, we can limit ourselves to the situation where an individual BGP router locally balances traffic over multiple paths, without changing BGP semantics.

We will first outline the modifications to BGP, after that prove loop-freeness, address convergence and finally briefly evaluate the result of these changes.

\section{MUltipath MOdificAtions to BGP}

For the past 15 years, the Border Gateway Protocol (BGP) version 4 [4] has been the only routing protocol used between the individual networks (routing domains or autonomous systems) that collectively make up the Internet. External BGP

This work has been partly funded by Trilogy, a research project (ICT-216372) supported by the European Community under its Seventh Framework Programme. The views expressed here are those of the author(s) only. The European Commission is not liable for any use that may be made of the information in this document. 
(eBGP) sessions are configured between routers located at each side of the border between two ASes, while BGP routers within an AS communicate through iBGP sessions. BGP is much like a distance vector routing protocol, but rather than using a simple hop count or cost metric, it uses a list of the AS numbers of all the ASes between the local AS and a destination (stored in the AS_PATH attribute) to suppress loops. Potentially, for every possible destination, a router learns a path to that destination from several neighbouring BGP routers. The BGP protocol then selects a best path by computing a degree of preference for all paths to a given destination received from BGP speakers in neighbouring routing domains, and then selects the path with the highest degree of preference (expressed in the LOCAL_PREF attribute). BGP specifically employs seven tie breaking rules to end up with a single path towards each destination when there are multiple paths with a same LOCAL_PREF value.

Multipath operation is not part of the BGP specification and not enabled by default, but many vendors like Cisco [5] or Juniper [6] allow multiple BGP paths/routes to be inserted in a router's routing table, each pointing towards a different next hop address. Each of these multiple routes is then used to send a fraction of the packets to the destination.

Like the solution proposed in this article, Cisco's solution respects BGP semantics. It is, however, too restrictive with the conditions that a path must fulfil in order to be selected (extra paths are almost equal to the best one). Juniper's solution is oriented more towards the provisioning of backup links and load balancing between adjacent BGP peers.

In order to utilise multiple paths towards a destination, we follow the BGP path selection rules, in particular the rule that only paths that share the highest LOCAL_PREF are selected. This makes sure that existing policies remain applicable, with one exception. We remove the tie breaking rules, including the selection of only those paths that share the shortest AS PATH length. However, there are additional rules as outlined below that govern which paths may be used to forward packets and that determine which of those paths is disseminated to neighbours.

We use the following notation:

$\pi$ the set of paths towards a destination disseminated to the local router by neighbouring routers

$P$ the set of paths towards a destination that are under consideration for being used

$P^{e}$ paths to a destination learned from neighbours in adjacent routing domains (eBGP)

$P^{i}$ paths to a destination learned from neighbours in the local routing domain (iBGP)

$R$ the set of neighbouring routers

$p_{r}$ the path selected for dissemination

$a_{p}$ AS_PATH length for path $p$

$c p(p)$ the cost to reach a destination through path $p$

$c p_{r}(p)$ the cost to reach a destination through path $p$ that is reported to other routers

$c(x)$ the cost to reach destination $x$

$c_{r}(x)$ the cost to reach destination $x$ that is reported to other routers

$\operatorname{set}(p)$ TRUE if the AS_PATH of $p$ contains an AS_SET, FALSE otherwise

\section{A. Too Many Paths}

Removing the tie breaking rules has the potential to create a set of usable paths that is too large to be workable. However, many of the expected benefits can be achieved with a small amount of choices [7]. Depending on hardware limitations, it may be desirable to limit the number of paths by executing the tie breaking rules until the number of paths meets a predetermined maximum.

\section{B. Low-quality Paths}

Although the AS_PATH length is not an accurate metric of a path's quality, completely disregarding the AS_PATH length may result in selecting inferior paths, as paths with very long AS_PATHs do tend to be inferior to those with short AS_PATHs. But only accepting paths that have an equal AS_PATH length limits the number of usable paths without good reason. Also, since we need to disseminate the path with the longest AS_PATH to upstream ASes, selecting paths with very long AS_PATHs will lead upstream ASes to prefer alternate downstream ASes, which would be detrimental for commercial network operators.

Considering this, we will use value $\alpha$ as the difference in AS_PATH length that is allowed between the path with the shortest AS_PATH and the path with the longest AS_PATH. For example, if $\alpha$ is 1 and the shortest AS_PATH among the paths in $P$ is 2 ASes, then paths with an AS_PATH length of 3 will be accepted in $P$ but not paths with an AS_PATH length of 4 . We will discuss different choices for $\alpha$ in the evaluation section.

Paths with too long AS_PATHs are removed from $P$ :

$$
\begin{aligned}
& \begin{array}{l}
a_{p}>a_{p}+\alpha \Rightarrow\{P\} \backslash p \\
\qquad \forall p \in P, \forall q \in P, p \neq q
\end{array} \\
& \begin{array}{l}
\text { C. Avoiding Suppression of eBGP Paths Towards iBGP } \\
\quad \text { Neighbours }
\end{array}
\end{aligned}
$$

\section{Avoiding Suppression of eBGP Paths Towards iBGP Neighbours}

A central notion to BGP is that a router only disseminates the path that it uses itself for forwarding packets. With iBGP, there is the additional limitation that a router may only disseminate a path that is learned over eBGP or generated locally. Disseminating paths learned through iBGP over iBGP may introduce loops.

A router can either be a source or a sink of packets towards a given destination relative to other routers within the local AS. If the router uses one or more iBGP-learned paths to reach a destination, it acts as a source and it cannot disseminate any paths of its own over iBGP or packets will 
loop. Only when all paths in $P$ are eBGP-learned paths, a router can be a sink for that destination in the local AS and advertise a path over iBGP. This limitation is expressed in equation (11).

Given this limitation, it would be sub-optimal to accept low-quality iBGP-learned paths in $P$, as these make the router's eBGP paths unavailable for use by the rest of the AS. For this reason, we do not accept iBGP paths in $P$ that have an equal or longer AS_PATH than the shortest AS_PATH among eBGP paths in $P$ :

$$
\begin{aligned}
p \in P^{i} \wedge q \in P^{e} \wedge a_{p} \geq a_{q} \Rightarrow & \{P\} \backslash p \\
& \forall p \in P, \forall q \in P^{e}, p \neq q
\end{aligned}
$$

\section{LOOP-FREENESS}

Under normal circumstances, the BGP AS_PATH attribute guarantees loop-freeness. Since our changes allow BGP to use multiple paths concurrently, but only a single path is disseminated to neighbouring ASes, checking the AS_PATH for the occurrence of the local AS number is no longer sufficient to avoid loops. Instead, we depend on the Vutukury/Garcia-Luna-Aceves Loop-free Invariant (LFI) conditions [8].

Intuitively, these conditions are very simple: because a router can only use paths that have a lower cost than the path that it disseminates to its neighbours (or, may only disseminate a path that has a higher cost than the paths that it uses), loops are impossible. A loop occurs when a router uses a path that it disseminated earlier, in which case the path that it uses must both have a higher and a lower cost than the path that it disseminates, situations that can obviously not exist at the same time.

When the following two LFI conditions as formulated by Vutukury and Garcia-Luna-Aceves are satisfied, paths are loop-free:

$$
\begin{aligned}
& F D_{j}^{i} \leq D_{j i}^{k} \\
& S_{j}^{i}=\left\{k \mid D_{j k}^{i}<F D_{j}^{i} \wedge k \in N^{i}\right\}
\end{aligned} \quad k \in N^{i}
$$

"where $D_{j k}^{i}$ is the value of $D_{j}^{k}$ reported to i by its neighbour $k$; and $F D_{j}^{i}$ is the feasible distance of router i for destination $j$ and is an estimate of $D_{j}^{i}$, in the sense that $F D_{j}^{i}$ equals $D_{j}^{i}$ in steady state but is allowed to differ from it temporarily during periods of network transitions. " [8]. $D_{j}^{k}$ is the distance or cost from router $k$ to destination $j$. $N^{i}$ is the set of neighbours for router $i$ and $S_{j}^{i}$ is the successor set that router $i$ uses as next hop routers for destination $j$.

Our interpretation of the two LFI conditions as they relate to BGP is as follows:

$$
\begin{aligned}
& c p\left(p_{r}\right)<c p_{r}\left(p_{r}\right) \\
& P=\left\{p \mid c p(p) \leq c p\left(p_{r}\right) \wedge p \in \pi\right\}
\end{aligned}
$$

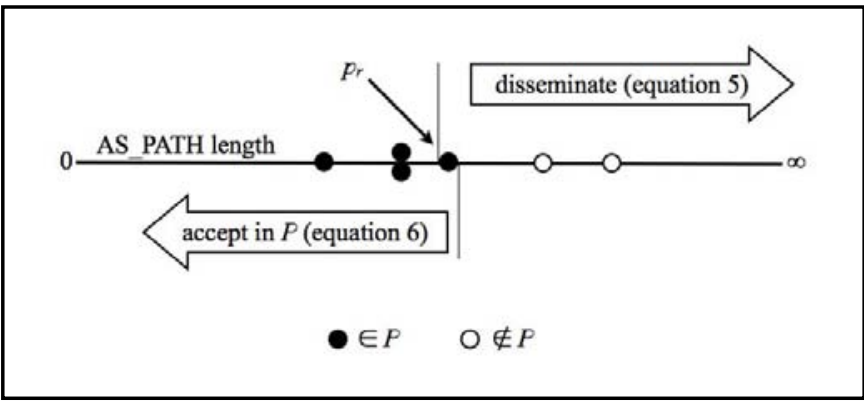

Fig. 1. The relationship between the cost (in this case, the AS_PATH length),

Where $c p(x)$ is taken to mean $a_{x}$ in the case of eBGP and the interior cost for iBGP. The interior cost is the cost to reach a destination as reported by the interior routing protocol that is in use. Because the local AS is added to the AS_PATH when paths are disseminated to neighbouring ASes, we swap the smaller and strictly smaller requirements between the two conditions. Fig. 1 shows the relationship between the cost (in this case, the AS_PATH length), $p_{r}$ and (5) and (6).

Equations (3) to (6) are not part of our modified BGP processing rules, as (5) and (6) are reformulations of (3) and (4), and (7) satisfies both LFI conditions for iBGP while (8) satisfies the LFI conditions for eBGP.

\section{A. Loop-freeness for $i B G P$}

Paths learned through iBGP may not be used if the interior cost towards the NEXT_HOP of the path is equal to or larger than the lowest interior cost towards a NEXT_HOP for paths from the multipath set as reported to other routers by the local router:

$$
c\left(h_{p}\right) \geq c_{r}\left(h_{q}\right) \Rightarrow\{P\} \backslash p \quad \forall p \in P^{i}, \forall q \in P
$$

This rule satisfies the requirement imposed by (6) for iBGP.

\section{B. AS_SETS}

The BGP-4 specification [4] allows for the aggregation of multiple prefixes into a single one. In that case, the AS numbers in the AS_PATH are replaced with one or more AS_SETs, which contain the AS numbers in the original paths.

Should the situation arise where a topology is not valleyfree [9] and there is both a router that implements multipath BGP as described in this paper as well as, in a different AS, a router that performs aggregation through the use of AS_SETs, then routing loops may be possible. This is so because, depending on the implementation, a router creating an AS_SET could shorten the AS_PATH length and break the limitations imposed by (5) and (6).

To avoid these loops, $P$ may either contain a single path with an AS_PATH that contains an AS_SET, or no paths with AS_PATHs that contain AS_SETs: 


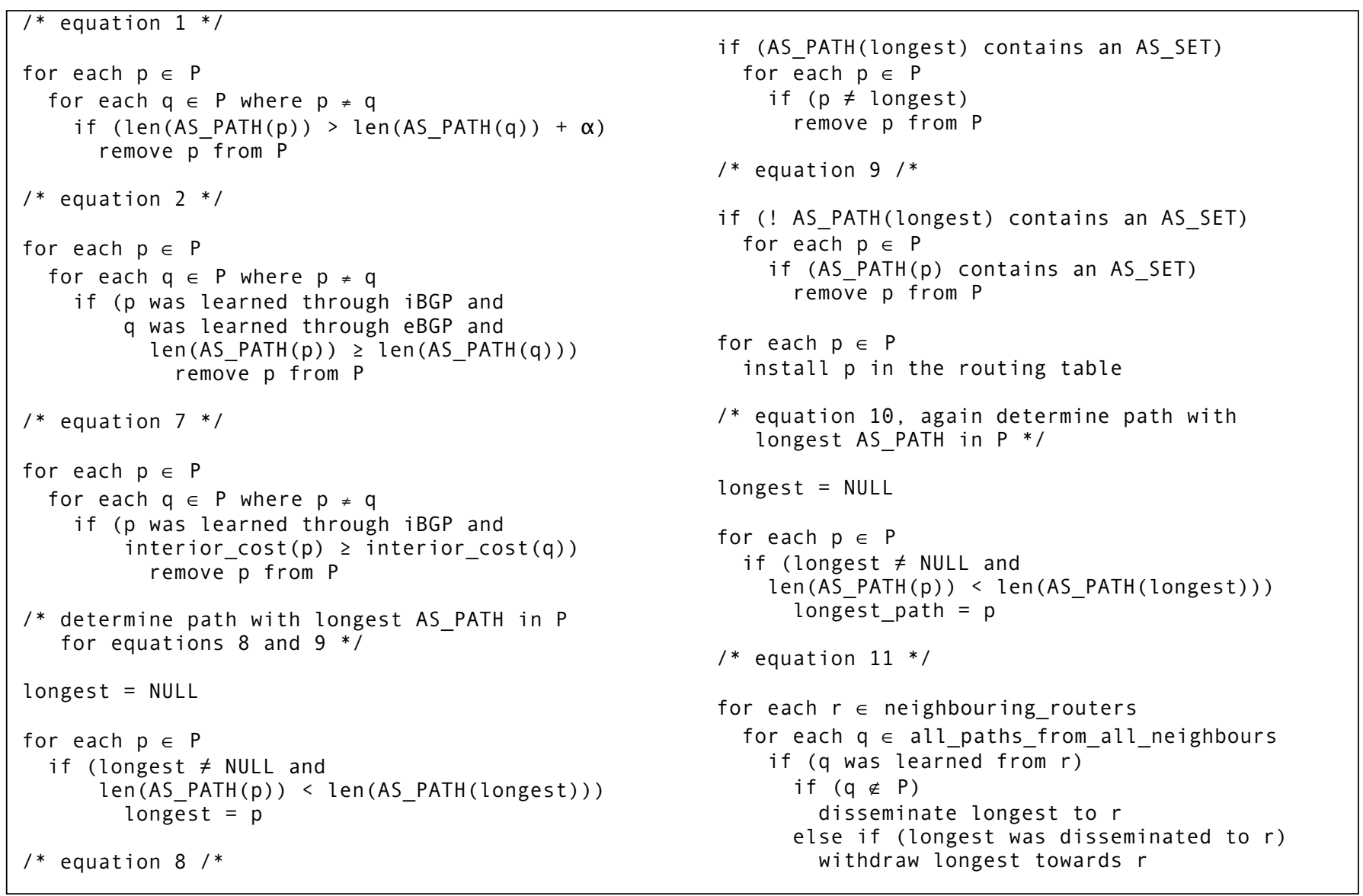

Fig. 2. The modified path selection and dissemination rules for multiptah BGP in pseudo-code.

$$
\begin{aligned}
& a_{p}=\max \left(a_{p}\right) \wedge \operatorname{set}(p) \Rightarrow P=\{p\} \quad \forall p \in P \\
& a_{p} \neq \max \left(a_{p}\right) \wedge \operatorname{set}(p) \Rightarrow\{P\} \backslash p \quad \forall p \in P
\end{aligned}
$$

Note that AS_SETs are rarely used today; a quick look through the Route Views project [10] data reveals that less than $0.02 \%$ of all paths have one or more AS_SETs in their AS_PATH.

\section{Disseminating Loop-free Paths in eBGP}

All paths that remain in the multipath set after the previous steps and after applying policy are installed in the routing table and used for forwarding packets. The determination of traffic split ratios between the available paths is a topic for future work.

At this point, the path with the longest AS_PATH within $P$ is selected for dissemination to BGP neighbours:

$$
a_{p}=\max \left(a_{p}\right) \Rightarrow p_{r}=p \quad \forall p \in P
$$

Equation (10) satisfies the requirement imposed by (5) for both iBGP and eBGP as well as the requirement imposed by (6) for eBGP. iBGP uses the interior cost, not the AS_PATH length, as its cost, so (10) does not address iBGP.

Through (10), multipath-aware ASes will suppress looped paths with a multipath-aware AS in the looped part of the path, while regular BGP AS PATH processing suppresses looped paths with no multipath-aware ASes in the looped part of the path.

If multiple paths share the maximum AS_PATH length, the path that was previously disseminated to BGP neighbours, if any, is selected for dissemination. This has the effect of damping oscillations on shorter paths.

\section{Loop-freeness for Multipath-unaware iBGP Routers}

To avoid loops for non-multipath-aware iBGP routers, the selected path is also not disseminated over any BGP session through which the router learned a path that is in the multipath set:

$$
\begin{aligned}
& q \notin P \Rightarrow \text { disseminate } p_{r} \\
& \begin{array}{l}
q \in P \Rightarrow \text { withdraw / do not disseminate } p_{r} \\
\qquad \forall r \in R, \forall q \in \pi, r=r_{q}
\end{array}
\end{aligned}
$$

If the router previously disseminated a path over a session towards a neighbouring router that supplied a path in the selected multipath set $P$, it now sends a withdrawal for the multipath destination.

Figure 2 shows pseudo-code that implements the new 


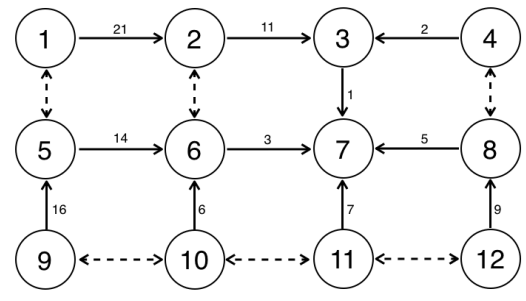

Fig. 3. Preferred unmodified BGP reachability and backup paths towards AS 7 .

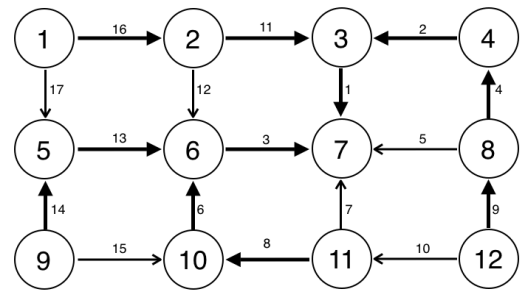

Fig. 4. Preferred multipath reachability towards AS $7, \alpha=\infty$

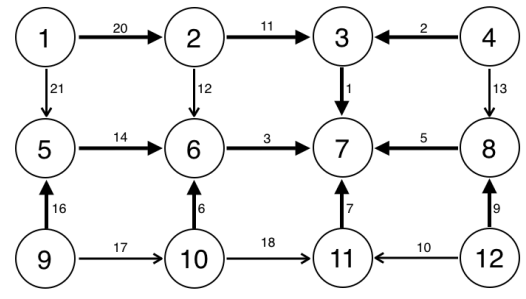

Fig. 5. Preferred multipath reachability towards AS $7, \alpha=1$ multipath BGP path selection and dissemination rules.

\section{CONVERGENCE}

Intuitively, it is easy to see that BGP topologies with conflicting policies have trouble converging [11]. For instance, if A prefers to send traffic through B, while B prefers to send traffic through A, BGP's loop detection will make sure that both do not happen at the same time, but it will not be possible to reach a stable, converged state: the final state depends on the order of events.

On the other hand, when the Gao/Rexford valley-freeness guidelines are observed [9], convergence to a stable state is guaranteed because in that case, there are no cycles in the configured policies. This means that whenever an AS selects a path, decisions made subsequently by upstream ASes will not make the earlier AS select a different path.

Like standard BGP, our path selection rules require that policies are such that only paths with the highest LOCAL_PREF are included in the candidate route set $P$. Because each LOCAL_PREF value maps to a single valleyfree class (sibling, service provider, peer or customer), our use of multiple paths does not break the valley-free property if it was present in the single path case.

So, in the case of valley-free topologies, eventual convergence is guaranteed and largely the same as that for a single path topology where only the longest paths from the equivalent multipath topology exist. However, there are more intermediate states and updates for those states, which may trigger the minimum route advertisement interval, pushing convergence times towards the maximum imposed by this interval. Non-valley-free topologies may never converge. The presence of longer paths injected by multipath-aware routers may exacerbate this situation as the multipath-aware routers try to find the longest loop-free paths allowed by policy. The quantification of these effects is part of our future work.

\section{Evaluation}

In order to evaluate the impact of our changes to BGP, in particular to get a grasp on the dynamics of the resulting system, we created a simulator that implements our modified BGP rules [12]. The simulator is a script that outputs the result of (multipath) BGP decision making based on a given input topology. The script can also simulate the decision making in traditional BGP routers. Figure 3 shows an example topology with all the routers using the existing BGP path selection and tie breaking rules. Each circle is an AS with a single router in it. Only the router in AS 7 announces a prefix. The solid arrows indicate the path selected by each AS, with the dotted arrows indicating additional paths present in the BGP table but not used. Note that in each case, unused paths exist in both directions.

The numbers adjacent to the arrows are the iteration numbers for the iteration when this path was selected. Note that the definition of iteration for this purpose is such that only a single router makes a path selection decision about a single path; in reality many decisions are made in parallel due to the distributed nature of the Bellman-Ford algorithm. Figure 4 shows the same topology as figure 3, but now all routers are multipath enabled and $\alpha$ is set to infinity. Each arrow indicates a path used for forwarding packets, the heavy arrow indicates the path disseminated to neighbours.

Interestingly, the number of iterations needed for multipath BGP to converge is actually slightly lower than the number of iterations needed by traditional BGP. This is probably because each router greedily obtains all the paths that it can, limiting the choices of other ASes.

Figure 5 shows the same topology with multipath BGP enabled, but now with a value of 1 for $\alpha$, so a router will not select any paths that have an AS_PATH that is more than one AS hop longer than the shortest available AS_PATH. In this case, only paths with equal length AS_PATHs are selected. In this case, AS 12 selects path $8-\overline{7}$ as the path that it disseminates to neighbours while in figure 4 , this was the path 8-4-3-7. So excessively long paths are avoided, while half of the ASes are still capable of using a second path. However, the number of iterations required to converge is back to 21, the same as traditional BGP.

While much further understanding of the dynamics is needed, the obtained results are promising, since the results from the experiments performed show that the proposed multipath BGP converges in a similar (if not smaller) number of iterations as current BGP and that it manages to avoid long paths, all this in challenging topologies.

\section{RELATED WORK}

The most common multipath mechanisms are the ones 
existing for intra-domain protocols, like the ECMP (Equal Cost Multipath) in OSPF [2] or IS-IS, the unequal cost multipath in IGRP and EIGRP [3] or provided by means of basic source routing mechanisms. All these proposals to select multiple paths inside an AS may work together with the solution proposed in this paper, which is mainly issued for inter-domain routing.

Some multipath proposals for inter-domain routing follow the intra-domain alternatives like the source routing proposals in [13] or [14] or the proposal based on overlays like MIRO [15]. As discussed in [15], the source routing proposals are in general too restrictive for the intermediate ASes whose flexibility to decide on alternative routes is reduced. Regarding the overlay solutions, they normally imply an additional complexity associated with the tunnelling mechanisms and the overhead that the tunnels introduce.

MIRO, however, reduces the overhead during the path selection phase by means of a co-operative path selection involving the different intermediate ASes (additional paths are selected on demand rather than disseminating them all every time). The proposal made in this paper does not require an overlay for the multipath mechanism to work, making deployment easier, since it does not require changes in the neighbouring ASes.

\section{CONCLUSION AND FUTURE WORK}

Multipath inter-domain routing is a powerful tool that results in substantial advantages, including increased network capacity, enhanced redundancy and better response to congestion events. We have shown that, contrary to the limitations accepted in common practice, it is possible to accept multiple paths for forwarding packets without risk of routing loops. This can be achieved without changes in the BGP semantics and only requiring local changes in the BGP route processing mechanism. This results in a powerful deployment model based in the incentive vector where the party that deploys the mechanism is the party that gets the benefits. There is no need for other ASes to also implement multipath BGP.

Our modifications can be applied transparently and incrementally by network operators, with only two effects that could be considered undesirable. Like existing multipath implementations, as far as those allow paths with different AS_PATH attributes to be used concurrently, only one of the paths used for transmitting packets is visible to upstream ASes; the contents of the AS_PATHs of other paths cannot be used for the application of policy. In addition, the propagation of a longer path could lead upstream ASes to select to select a different path and no longer send traffic.

Additional research is needed to fully understand the impact of the proposed mechanism. First, the resulting dynamics of the proposed BGP multipath approach need further investigation. In particular, even if we know that the proposed modification does not change the convergence result (i.e. configurations that converge in regular BGP still converge in our proposed multipath approach), additional analysis is required in how the proposed changes affect the convergence process, including a quantification of the expected number of iterations to converge. In addition, we need to quantify the increase in the stability of the resulting paths. As we mentioned before, shorter path changes are no longer propagated, so there is potential reduction in routing churn that needs to be quantified.

Another aspect that needs more research is the resulting path distribution and diversity if the proposed mechanism is widely implemented.

Other approaches to multipath BGP would be to disseminate AS_SETs containing all the ASes in AS_PATHs of all paths used, and changing BGP such that multiple paths can be communicated between two neighbours.

\section{REFERENCES}

[1] F. Kelly, T. Voice. "Stability of end-to-end algorithms for joint routing and rate control". ACM SIGCOMM Computer Communication Review. vol. 35 , no. 2 , pp. 5-12, 2005.

[2] J. Moy. “OSPF Version 2". RFC 2328, April 1998.

[3] R. Albrightson, J.J. Garcia-Luna-Aceves, and J. Boyle. "EIGRP-A Fast Routing Protocol Based On Distance Vectors", in Proc. Networld/Interop Conf, Las Vegas, Nevada, USA, 1994, pp. 136-147.

[4] Y. Rekhter, T. Li, S. Hares. "A Border Gateway Protocol 4 (BGP-4)". RFC 4271, January 2006.

[5] BGP best path selection algorithm. Document ID: 13753. May 2006. Cisco Systems. [Online]. Available: http://www.cisco.com/application/pdf/paws/13753/25.pdf.

[6] J. M. Soricelli. Juniper ${ }^{\mathrm{TM}}$ Networks Certified Internet Specialist Study Guide. Sybex, 2004.

[7] M. Mitzenmacher. "The Power of Two Choices in Randomized Load Balancing". PhD Thesis, University of California, Berkeley, California, 1996.

[8] S. Vutukury, J.J. Garcia-Luna-Aceves. "A Simple Approximation to Minimum-Delay Routing”, in Proc. ACM SIGCOMM Conf, Cambridge, Massachusetts, USA, 1999, pp. 227-238.

[9] L. Gao, J. Rexford. "Stable Internet Routing Without Global Coordination", in Proc. ACM SIGMETRICS Conf, Santa Clara, California, USA, 2000, pp. 681-692.

[10] Route Views project [Online]. Available:http://www.routeviews.org, 2008.

[11] K. Varadhan, R. Govindan, D. Estrin. "Persistent route oscillations in inter-domain routing". ISI technical report 96-631, USC/Information Sciences Institute, 1996.

[12] I. van Beijnum. "runbgp script and example topologies" [Online]. Available: http://www.bgpexpert.com/runbgp.tar.gz. September 2008.

[13] D. Zhu, M. Gritter, D. Cheriton. "Feedback based routing", in Proc. Hotnets Workshop, Princeton, New Jersey, USA, 2002, pp. 71-76.

[14] H. T. Kaur, S. Kalyanaraman, A. Weiss, S. Kanwar, A. Gandhi. "BANANAS: An evolutionary framework for explicit and multipath routing in the Internet", in Proc. Future Directions in Network Architecture Conf, Karlsruhe, Germany, 2003, pp. 277-288.

[15] W. Xu, J Rexford. "MIRO: multi-path interdomain routing", in Proc. ACM SIGCOMM Conf, Pisa, Italy, 2006, pp. 172-182 